\title{
Malaria elimination practices in rural community residents in Rwanda: A cross sectional study
}

\author{
Domina Asingizwe ${ }^{1 *}$, Stephen Rulisa ${ }^{1,2}$, Brenda Asiimwe-Kateera ${ }^{1}$, Mi Ja Kim ${ }^{1}$ \\ ${ }^{1}$ University of Rwanda, College of Medicine and Health Sciences \\ ${ }^{2}$ Department of Obstetrics and Gynecology and Department of Research, University Teaching Hospital of Kigali.
}

\begin{abstract}
Background: Rwanda is moving towards malaria pre-elimination phase by the year 2017 and the role of the community will be critical. However, there is limited information about community perspective of the malaria elimination strategy. A study was thus designed to explore that. Methods: A descriptive cross-sectional study was conducted using a validated questionnaire. A total of 150 community residents from 10 villages in a high endemic area in Bugesera district, Eastern Province, Rwanda, participated in this study. Multi stage sampling was used to select households. Results: Knowledge and attitude were main factors contributing to malaria elimination practices. Most participants knew that the mosquito was the vector which transmits malaria $(90.7 \%, \mathrm{n}=136)$; malaria was transmitted to humans by bites of mosquito infected with malaria $(90.7 \%, \mathrm{n}=136)$. Most key signs and symptoms as well as control measures for malaria were known to the participants. Their attitude about complete malaria elimination was highly positive $(90.7 \%, \mathrm{n}=136)$. Conclusion: The community in Bugesera area were quite optimistic of the malaria elimination targets and what is needed to be done is to support those who are not yet quite aware of the activities in this direction.
\end{abstract}

Key words: Malaria elimination practices, community, knowledge, attitude, Rwanda, Africa

\section{Background}

Malaria is a major public health concern in many parts of the world, especially in sub-Saharan Africa where about $90 \%$ of all malaria deaths occur (WHO, 2012). Malaria is an entirely preventable and treatable disease, provided that effective interventions are properly implemented (WHO, 2013).

The government of Rwanda has made significant achievements in scaling up malaria control interventions. During an intense 15-month campaign, Rwanda distributed over 6.1 million long lasting insecticide treated nets and was one of the first African countries to reach universal net coverage in February 2011 (Mazigo et al., 2010a). Since 2008, there was a decrease in malaria morbidity and mortality with a drastic drop in Malaria attributed mortality rate from $16.3 \%$ in 2008 to $3.6 \%$ in 2011 (President's Malaria Initiative, 2013). However, there has recently been an increase in malaria prevalence which is a cause of concern. Rwanda has five high malaria burden districts which accounted for $71.6 \%$ of the malaria burden in 2011 and Bugesera was classified as the second, these include Nyagatare $41.7 \%$, Bugesera 8.4\%, Rusizi 7.8\% Kirehe 7.5\% and Gisagara 6.2\% (President's Malaria Initiative, 2013). In 2013, there was still a high prevalence of malaria $(22.8 \%)$ among health centre attendees and 5.1\% within the households members in Bugesera district (Rulisa et al., 2013). Poor involvement of the community in malaria elimination, misuse of mosquito nets, and misconceptions of malaria preventive measures were cited as problems among community residents, which hindered the success of malaria elimination (Ingabire et al., 2014).

Elimination of malaria from a community requires a combination of several factors including comprehensive knowledge, positive attitudes, improved sanitation in the home environment including rice farming, removal of stagnant water and bushes, sleeping under mosquito nets and participation in malaria elimination measures (AlAdhroey, Nor, Al-Mekhlafi, \& Mahmud, 2010). Socio demographic factors such as education and gender are identified as important determinants that influenced practice of malaria elimination measures, especially related to mosquito nets possession and usage. (Mazigo et al., 2010a). Limited use of insecticide treated nets and close location of homesteads in relation to breeding sites have been recognized as risk factors for malaria transmission (Chirebvu, Chimbari, \& Ngwenya, 2014) In Sub Saharan Africa, numerous studies (Abate, Degarege, \& Erko, 2013; Chirebvu, Chimbari, \& Ngwenya, 2013; Opare et al., 2014; Spjeldnæs, Kitua, \& Blomberg, 2014) have reported on community residents' knowledge about the cause, signs and symptoms,

*Corresponding author: adomina@khi.ac.rw 
transmission and preventive methods of malaria. Many studies in Rwanda have reported about the prevalence and risk factors related to malaria, and community mobilisation for malaria elimination as well as factors impeding the acceptability and the use of malaria preventive measures (Gahutu et al., 2011; Ingabire et al., 2014; Ingabire et al., 2015; Kateera et al., 2015; Rulisa et al., 2013). However, there is limited information about the knowledge and attitude of community residents towards malaria elimination. Hence, this study aimed to describe the key factors that contribute to malaria elimination practice; describe strategies used by community residents to prevent malaria; and analyse the relationship between knowledge and attitude towards malaria elimination and malaria elimination practices.

\section{Methods}

\section{Study area}

This study was carried out in Bugesera district, Eastern Rwanda which is predominantly rural and highly endemic for malaria. It is composed of 15 sectors, 72 cells and 581 villages with a total population of 363,336 people. In 2011, indoor residual spraying (IRS) coverage was 98.6\%.

\section{Sampling procedures}

This study used a multistage sampling method to obtain a sample of 150 people. First stage involved simple random selection of three sectors (Rilima, Mareba and Rweru) out of 15 sectors in Bugesera district; the second stage involved random selection of one village in each selected sector (Amizero, Kururama and Nzangwa villages). The third stage was to conveniently select three additional villages neighbouring the first village in Rilima and Mareba Sectors and one village neighbouring the first village in Rweru Sector. In each village, 15 households were selected by convenient sampling.

\section{Study design and participants}

A descriptive cross sectional study was done. From ten villages in Bugesera district, 150 households were selected. Using a structured questionnaire, interviews were conducted in Kinyarwanda with the head of the household or a responsible adult member if the head of household was not available.

\section{Measurements and data collection procedure}

A closed ended structured questionnaire was translated and back translated from English to Kinyarwanda using consensus approach. The questionnaire had 42 closed ended questions with single or multiple responses and
Likert scale. Content validity was established through conceptualization of the construct, which was carried out by extensive literature review and consultation with five experts who are familiar with development, adaptation and validation of malaria related questionnaires as well as conducting and coordinating the malaria related studies. To ensure its reliability, the questionnaire was tested twice with 10 community residents from Ntarama sector which was not one of the study sites in Bugesera district. The test-retest coefficient was 0.82 . A one-day training about data collection procedures and research ethics including the informed consent process was conducted for a registered nurse who collected data.

Questionnaires were completed by study staff based on the interviewees' responses; and cross-checked with the participants at the end of the interviews for accuracy and completeness. The principal investigator checked all the questionnaires at the end of each day for completeness.

\section{Scoring}

The scores were used in order to categorize knowledge (poor or good), attitudes (negative or positive) and practices (poor or good). For determining knowledge scores, the following four multiple-choice questions were used; 1. Vector which transmit malaria, 2, Mode of malaria transmission, 3. Untreated malaria can kill and 4. The most way used for malaria prevention, control and elimination. Each of the questions scored one point for a correct response and zero for an incorrect response. An overall knowledge score was calculated by adding up the scores for each respondent across all questions. Thereafter, those answered all questions correctly (having four points) were considered as having good knowledge and others were taken as having poor knowledge. A similar scoring was used for attitudes. Six questions/ statements (table 2) that indicate malaria attitude were scored for each respondent. If a respondent answered correctly the statement they were given a score of four points. If not they were given a score of one point. An overall attitude score was determined for each respondent by adding up the scores across the malaria attitude questions. Thereafter, those answered all questions correctly were considered as having positive attitudes and others were taken as having negative attitudes. For the practices, seven questions (table 3 ) that indicate malaria elimination practices were scored for each respondent. If a respondent indicated that he/she always performed a good practice they were given a score of two points. If they indicated that they sometimes performed a good practice, they were given a score of one point. On the other hand, if they indicated they never performed a good practice, they were given a score of zero. An overall practices score was determined for each respondent by 
adding up the scores across the eight malaria practices questions. Thereafter, those answered at least 5 questions correctly (said "always") were considered as having good practices and others were taken as having poor practices.

\section{Data Analysis}

Data were analysed using Stata version 13. Descriptive statistics using frequencies and percentages were used. The main outcome was malaria elimination practices which included sleeping under an insecticide treated mosquito nets, cleaning the home environment, draining stagnant water, closing the windows and treatment seeking behaviour.

Bivariate and multivariate analyses were done to examine the relationship between malaria elimination practices and socio-demographic variables, knowledge and attitude towards malaria elimination. The level of significance was set at an alpha of 0.05 .

\section{Ethical Issues}

Mount Kenya University Institutional Review Board approved the study protocol and the Mayor of Bugesera district gave permission to conduct the study. Before study initiation, health centres and community health care workers in the study catchment area were informed about the study. Signed written consent forms were obtained from each head of household or adult member. The principles of privacy, confidentiality and voluntarism were upheld.

\section{Results}

\section{Demographic characteristics of the respondents}

One hundred fifty (150) heads of household or adult member participated in the study. The majority of the respondents were female $(70 \%, \mathrm{n}=105)$. The mean age of the respondents was 37.2 years (standard deviation $12.8)$ and $(65 \% \mathrm{n}=98)$ were married. Over half $(52 \%$, $\mathrm{n}=78$ ) had not completed primary school or had no formal education; only $(6.7 \%, \mathrm{n}=10)$ respondents reported completing secondary school education. Most of the families had four or five $(41.3 \%, \mathrm{n}=62)$ or six or more household members $(31.3 \%, \mathrm{n}=47)$.

\section{Malaria knowledge}

Most of the respondents $(90.7 \%, n=136)$ knew that malaria was transmitted to humans by bites of mosquitoes infected with malaria. The reported signs and symptoms of malaria were fever $(92.7 \%, \mathrm{n}=139)$, headache $(70.7 \%$, $\mathrm{n}=106)$, shivering $(18.7 \%, \mathrm{n}=28)$, sweating $(17.3 \%$, $\mathrm{n}=26)$ and $(11.3 \%, \mathrm{n}=17)$. Regarding knowledge related to malaria elimination practices, use of insecticide treated mosquito nets $(96.7 \%, n=145)$ was identified as the most effective way followed by elimination of bushes in the home environment $(71.3 \%, \mathrm{n}=107)$. Although knowledge related to malaria transmission, signs and symptoms of malaria, and malaria practices seemed to be good in this area, $(43 \%, n=64)$ reported that they did not have adequate information related to malaria and its elimination. Of these, $(47 \%, \mathrm{n}=30)$ indicated the need to be given information related to elimination of malaria.

Table 1. Knowledge regarding causes, transmission and preventive methods of malaria

\begin{tabular}{lll}
\hline Variables & Category & Frequency (\%) \\
\hline Causes of malaria mentioned* & Mosquito bite & $136(90.7)$ \\
& Unclean place & $27(18)$ \\
& Contaminated water & $20(13.3)$ \\
\hline Mode of transmission & Bite of mosquito infected with malaria & $136(90.7)$ \\
& Through drinking unclean water & $4(2.6)$ \\
\hline Signs/symptoms of malaria* & By flies from dirty place & $10(6.7)$ \\
& Fever & $139(92.7)$ \\
& Headache & $106(70.7)$ \\
& Shivering & $28(18.7)$ \\
& Sweating & $26(17.3)$ \\
& Diarrhea & $17(11.3)$ \\
\hline Preventive methods* & Use of insecticide treated nets & $145(96.7)$ \\
& Elimination of bushes in the home environment & $107(71.3)$ \\
& Draining stagnant water & $106(70.7)$ \\
\hline Untreated malaria can kill & House spray with insecticide & $42(28)$ \\
\hline Has adequate information about malaria and its & Yes & $149(99.3)$ \\
elimination & No & $1(0.7)$ \\
\hline Information needed by the respondents & No & $86(57)$ \\
& Information about malaria elimination & $64(43)$ \\
\hline
\end{tabular}

*Multiple responses were allowed 


\section{Attitudes towards malaria elimination}

Majority of the respondents recognised the threat posed by malaria; $(96 \%, \mathrm{n}=144)$ believed that sleeping under mosquito nets was the most effective way for malaria elimination. Most participants $(96.7 \%, n=147)$ had positive attitudes toward treatment seeking behaviour and these included early diagnosis and treatment. (90.7\%, $\mathrm{n}=136$ ) of the participants viewed malaria elimination as an achievable target and they believed that their participation was essential to achieve that target.

Table 2. Attitudes toward malaria elimination $(\mathrm{N}=150)$

\begin{tabular}{lll}
\hline \multirow{2}{*}{ Characteristics } & \multicolumn{2}{c}{ Scores } \\
\cline { 2 - 3 } & Disagree N (\%) & Agree N (\%) \\
\hline I believe that sleeping under a insecticide treated net every night is the best way to & $6(4)$ & $144(96)$ \\
prevent malaria & & $145(96.7)$ \\
I think that it is dangerous when anti-malarial medication is not taken completely & $5(3.3)$ & $144(96)$ \\
I think that I should go to the health centre/clinic for a blood test as soon as I & $6(4)$ & $145(96.7)$ \\
suspect that I have malaria & $5(3.3)$ & $149(99.3)$ \\
I will seek for advice or treatment when I get malaria & $1(0.7)$ & $136(90.7)$ \\
\hline I think my engagement in malaria elimination is essential & $14(9.3)$ & I think with my participation and involvement, malaria elimination is achievable
\end{tabular}

\section{Malaria elimination practices}

The majority of the respondents $(83.3 \%, \mathrm{n}=125)$ indicated they always slept in insecticide treated mosquito net (ITN), however $(24.7 \%, \mathrm{n}=37)$ of household members never slept under an insecticide treated mosquito net. On the other hand also, almost half of the respondents $(46 \%$, $n=69)$ never checked for the holes or repaired mosquito nets even though they slept under mosquito nets.

Table 3. Malaria elimination practices $(\mathrm{N}=150)$

\begin{tabular}{|c|c|c|c|}
\hline \multirow[t]{2}{*}{ Variables } & \multicolumn{3}{|c|}{ Scores } \\
\hline & Never $(\%)$ & Sometimes (\%) & Always ( \%) \\
\hline How often do you sleep in a insecticide treated net? & $8(5.3)$ & $17(11.3)$ & $125(83.3)$ \\
\hline $\begin{array}{l}\text { How often do other members of the household } \\
\text { sleep in insecticide treated net? }\end{array}$ & $37(24.7)$ & $21(14)$ & $92(61.3)$ \\
\hline $\begin{array}{l}\text { How often do you check for holes/repair insecticide } \\
\text { treated net? }\end{array}$ & $69(46)$ & $23(15.3)$ & $58(38.7)$ \\
\hline $\begin{array}{l}\text { How often do you clean/cut bushes around your } \\
\text { house? }\end{array}$ & $4(2.7)$ & $22(14.7)$ & $124(82.7)$ \\
\hline $\begin{array}{l}\text { How often do you clean stagnant water near your } \\
\text { house? }\end{array}$ & $2(1.3)$ & $20(13.3)$ & $128(85.3)$ \\
\hline How often do you close the windows before $6 \mathrm{pm}$ ? & $1(0.7)$ & $31(20.7)$ & $118(78.7)$ \\
\hline $\begin{array}{l}\text { How often do you visit the health centre when you } \\
\text { fall sick? }\end{array}$ & $5(3.3)$ & $8(5.3)$ & $137(91.3)$ \\
\hline
\end{tabular}

Association between malaria elimination practices and age, gender, educational level, knowledge and attitude

Educational level, knowledge and attitudes related to malaria showed statistically significant association ( $p$ value $=0.049)$. It showed that respondents who had less education were more likely to have poor malaria practices compared to those having more education as well as those having positive knowledge $(\mathrm{p}$ value $=0.029)$ and positive attitudes $(\mathrm{p}$ value $=0.001)$. There was no association between malaria practices and the respondent's gender $(\mathrm{p}$ value $=0.146$ or age $(p$ value $=0.7)$. In the multivariate analysis, participants with at least primarily school education had $68 \%$ higher odds of having better malaria elimination practices compared to those with no primary or formal education, though this did not reach statistical significance. (OR=1.68, 95\%CI: .69-4). Similarly having high knowledge was positively associated with better malaria elimination practices $(\mathrm{OR}=1.82,95 \% \mathrm{IC}: .67$ 4.93). Age and gender were not associated with malaria elimination practices. 
Table 4. Association of malaria elimination practices with demographic variables, knowledge and attitudes

\begin{tabular}{|c|c|c|c|}
\hline \multirow{2}{*}{ Variables } & \multicolumn{2}{|c|}{ Malaria elimination practices } & \multirow{2}{*}{$P$ value } \\
\hline & Good practice & Poor practice & \\
\hline \multicolumn{4}{|l|}{ Age } \\
\hline$\leq 35$ years & $66(80.5)$ & $16(19.5)$ & \multirow{2}{*}{0.701} \\
\hline$>35$ years & $53(78)$ & $15(22)$ & \\
\hline \multicolumn{4}{|l|}{ Gender } \\
\hline Male & $39(86.7)$ & $6(13.3)$ & \multirow{2}{*}{0.146} \\
\hline Female & $80(76.2)$ & $25(23.8)$ & \\
\hline \multicolumn{4}{|l|}{ Education level } \\
\hline No or incomplete primary & $57(73)$ & $21(27)$ & \multirow{2}{*}{0.049} \\
\hline Primary and above & $62(86.1)$ & $10(13.9)$ & \\
\hline \multicolumn{4}{|l|}{ Knowledge } \\
\hline Low & $18(64.3)$ & $10(35.7)$ & \multirow{2}{*}{0.029} \\
\hline High & $101(82.8)$ & $21(17.2)$ & \\
\hline \multicolumn{4}{|l|}{ Attitude } \\
\hline Negative & $0(0)$ & $3(100)$ & \multirow{2}{*}{0.001} \\
\hline Positive & $119(81)$ & $28(19)$ & \\
\hline
\end{tabular}

Table 5. Multivariate anaylsis malaria elimination practices with demographic variables, knowledge and attitudes

\begin{tabular}{llll}
\hline Variables & Odds Ratio & P value & $\mathbf{9 5 \%}$ CI \\
\hline Gender & 0.5105594 & 0.214 & $0.1770325-1.472446$ \\
Age & 0.8831275 & 0.774 & $0.3779623-2.063471$ \\
Education & 1.682723 & 0.248 & $0.6962392-4.06693$ \\
Knowledge & 1.829075 & 0.233 & $0.6785079-4.930698$ \\
\hline
\end{tabular}

*Attitude was omitted due to the small number (3) of respondents who had negative attitudes

\section{Discussion}

This study aimed to describe factors that contribute to malaria elimination in a rural, highly malaria endemic area in Rwanda. Knowledge and attitudes were key factors. The participants' knowledge about causes, signs and symptoms and ways to prevent malaria was high. This corroborates the findings of a Tanzania study (Spjeldnæs et al., 2014) where most of participants had good knowledge about malaria prevention $(85.2 \%)$ and transmission (82.1\%). A similar study conducted in Botswana reported that the most of participants had knowledge related to preventive measures of malaria $(98.6 \%)$, malaria transmission (95.8\%) and its signs and symptoms $88.7 \%$ (Chirebvu et al., 2013). They are also similar to the findings of a study done in Colombia (Forero et al., 2014) where the knowledge related to malaria was found to be high. However, this is different from the study done in Ghana (Opare et al., 2014) where only few participants $(1.6 \%)$ could indicate the correct cause of malaria, and they showed fair (7.6-48.7\%) level of knowledge related to signs and symptoms of uncomplicated malaria. They differ also with the results of a study conducted by (Ingabire et al., 2014) in
Ruhuha sector of Bugesera district, Rwanda where some misconceptions of the cause of malaria and misuse of preventive strategies were noted. High level of knowledge demonstrated by our participants is encouraging given their low level of education. This may suggest that the community residents may have gained this knowledge through government programmes and other kinds of media.

Positive attitude demonstrated by participants in this study by sleeping in bed nets is similar to findings from Ethiopia that showed that the participants believed that mosquito nets protect from mosquito bite (Abate et al., 2013). The study conducted by (Karunamoorthi \& Kumera, 2010) indicated that the majority of the respondent visit health care facilities once they felt seek.

It is encouraging to note that the majority of the respondents always slept in a insecticide treated net $(83.3 \%)$. These results are in the same line with those found by (Ambrose, Mazigo, Heukelbach, Gabone, \& Mwizamholya, 2011) which indicated that $68 \%$ of the respondents reported they slept always under a bed net. They are also in agreement with the findings by (Mazigo et al., 2010b) where they indicated that 64.5\% 
of the respondents were using bed nets. However, $24.7 \%(n=37)$ of other household members never slept under an insecticide treated mosquito net. These results are in agreement with that of (Ingabire et al., 2014) who reported that some misconceptions and misuse of preventive measures were still observed in Ruhuha sector, Bugesera district, Rwanda. The results indicated that insecticide treated net may be related to having few compare to the number of beds that the household members were using or other misconceptions related to sleeping under insecticide treated net. The results also are quite different from that found by (Bamidele \& Ntaji, 2012) where only $23 \%$ used bed nets to protect themselves. While the high level of use of mosquito nets is good, one should be mindful of non-sleeping times that they did not use mosquito nets. It warrants our attention to a large number $(46 \%)$ of participants never checked for the holes or repaired holes in the mosquito nets. Improving overall sanitation of the environment and taking preventive measures should accompany their use of mosquito nets

Significant associations found between education level and malaria elimination practices, and between knowledge and attitude and malaria elimination practices are not surprising as they are closely related. A significant association between education and malaria practices demonstrated by participants in this study is similar to the findings from Tanzania, Nigeria and Ethiopia that showed that there was a strong association between educational status of the respondents and practice of malaria prevention and elimination measures (Adedotun, Morenikeji, \& Odaibo, 2010; Akinyede et al., 2011; Ambrose et al., 2011; Karunamoorthi \& Kumera, 2010; Spjeldnæs et al., 2014). Lack of significant association of gender and age with malaria elimination practices found in this study is different from the findings of a study done in Ghana (Opare et al., 2014) that showed that females were likely to know more about malaria prevention compared to males. Their findings of no relationship between educational levels and malaria elimination practices also are different from those of this study which indicated that respondents who didn't have primary level education were more likely to have poor malaria practices compared to those having primary level education and higher.

As a limitation, this study targeted the heads of household as a proxy to malaria elimination practices held by the household. The research team should have interviewed every adult in the household and also used observational check list, however, it was not possible due to constraints of resources. Questionnaire captured self reported information and relied primarily on respondents to provide right information. Accordingly, possibility of misreporting by the respondents cannot be ruled out.

\section{Conclusion}

Findings of this study showed high level of knowledge of the community residents about malaria, its signs and symptoms and preventive measures. The community residents used appropriate prevention strategies and had positive attitudes to malaria elimination. Malaria elimination practice had significant associations between education level, knowledge and attitudes. This suggests that raising general educational level of rural Rwandans beyond primary education can have needed. The gap found in checking for the holes or repairing mosquito nets indicates more focused education on the importance of maintaining the intact mosquito nets is crucial to prevent malaria. Judging from the big number of participants who indicated the need for more information related to malaria elimination measures, broader and more focused community sensitisation in rural areas of Rwanda are needed. The community health officers from health centres can play a big role in teaching the community about sleeping in mosquito nets always and regular checking whether the nets are intact. This would help achieve the Rwandan government's moving towards malaria pre-elimination phase by the year 2017. In spite of good knowledge and attitude to malarial elimination practices the problem of malaria has not been contained, therefore recommendations for further studies on change in working places, patterns and working times of residents, biting habits of mosquitoes etc. would be areas for further research.

\section{Competing interests}

The authors declare that they have no competing interests.

\section{Authors' contributions}

DA designed the study, coordinated fild activity, supervised data collection and drafted the manuscript. $\mathrm{SR}, \mathrm{MJK}$ and BK participated in drafting and revising the manuscript. All authors read and approved the final manuscript.

\section{Acknowledgements}

The acknowledgement goes to the IPMA (Integrated Research Partnerships for Malaria Control in Africa) for their funds that contributed to the success of this study. This work also was supported in-part by Africa 
Research Initiatives and Support Enterprise, grant number W.07.10.102 funded by Netherlands African Partnership for Clinical Trials and Capacity Building Program (NACCAP) of The Netherlands Organization for Scientific Research - The Netherlands Foundation for the Advancement of Tropical Research (NWO/ WOTRO). Its contents are solely the responsibility of the authors and do not necessarily represent the official views of the supporting office."

\section{References}

Abate, A., Degarege, A., \& Erko, B. (2013). Community knowledge, attitude and practice about malaria in a low endemic setting of Shewa Robit Town, northeastern Ethiopia. BMC Public Health, 13:312, 1-8. doi:10.1186/14712458-13-312

Adedotun, A. A., Morenikeji, O. A., \& Odaibo, A. B. (2010). Knowledge, attitudes and practices about malaria in an urban community in south-western Nigeria. Journal of Vector Borne Diseases, 47(3), 155-9. Retrieved from http://www. ncbi.nlm.nih.gov/pubmed/20834085

Akinyede, A., Akintonwa, A., Okany, C., Awodele, O., Olayemi, O. O., Dolapo, D. C., Yusue, A. (2011). Knowledge, treatment seeking and preventive practices in respect of malaria among patients with HIV attending Lagos University Teaching Hospital , Nigeria. Tanzania Journal of Health Research, 13(4), 1-11.

Al-Adhroey, A. H., Nor, Z. M., Al-Mekhlafi, H. M., \& Mahmud, R. (2010). Opportunities and obstacles to the elimination of malaria from Peninsular Malaysia: knowledge, attitudes and practices on malaria among aboriginal and rural communities. Malaria Journal, 9:137. doi:10.1186/14752875-9-137

Ambrose, E. E., Mazigo, H. D., Heukelbach, J., Gabone, O., \& Mwizamholya, D. L. (2011). Reported knowledge, attitudes and practices regarding malaria and mosquito net use among women seeking antenatal care in south-western Tanzania. Tanzania Journal of Health Research, 13(3), 1-9.

Bamidele J. O., Ntaji M. I., O. E. A. \& B. O. K. (2012). COMMUNITY PARTICIPATION IN MALARIA CONTROL IN OLORUNDA LOCAL, 6, 24-28.

Chirebvu, E., Chimbari, M. J., \& Ngwenya, B. N. (2013). Knowledge and practices on malaria in Tubu village, in a malaria - endemic area in northern Botswana : implications for interventions. Malaria World Journal, 4(15), 1-9.

Chirebvu, E., Chimbari, M. J., \& Ngwenya, B. N. (2014). Assessment of risk factors associated with malaria transmission in tubu village, northern botswana. Malaria Research and Treatment, 403069. doi:10.1155/2014/403069

Forero, D. A., Chaparro, P. E., Vallejo, A. F., Benavides, Y., Gutiérrez, J. B., Arevalo-Herrera, M., \& Herrera, S. (2014). Knowledge, attitudes and practices of malaria in Colombia. Malaria Journal, 13(165), 1-10. doi:10.1186/1475-2875-13165
Gahutu, J.-B., Steininger, C., Shyirambere, C., Zeile, I., CwinyaAy, N., Danquah, I., Mockenhaupt, F. P. (2011). Prevalence and risk factors of malaria among children in southern highland Rwanda. Malaria Journal, 10:134. doi:10.1186/14752875-10-134

Ingabire, C., Alaii, J., Hakizimana, E., Kateera, F., Muhimuzi, D., Nieuwold, I., Van Den Borne, B. (2014). Community mobilization for malaria elimination: application of an open space methodology in Ruhuha sector, Rwanda. Malaria Journal, 13:167. doi:10.1186/1475-2875-13-167

Ingabire, M. C., Rulisa, A., Van Kempen, L., Muvunyi, C., Koenraadt, C. J., Van Vugt, M., Alaii, J. (2015). Factors impeding the acceptability and use of malaria preventive measures: implications for malaria elimination in eastern Rwanda. Malaria Journal, 14:136. doi:10.1186/s12936-0150659-6

Karunamoorthi, K., \& Kumera, A. (2010). Knowledge and health seeking behavior for malaria among the local inhabitants in an endemic area of Ethiopia: implications for control. Health, 02(06), 575-581. doi:10.4236/ health.2010.26085

Kateera, F., Mens, P. F., Hakizimana, E., Ingabire, M. C., Muragijemariya, L., Karinda, P., van Vugt, M. (2015). Malaria parasite carriage and risk determinants in a rural population: a malariometric survey in Rwanda. Malaria Journal, 14:16, 1-11. doi:10.1186/s12936-014-0534-x

Mazigo, H. D., Obasy, E., Mauka, W., Manyiri, P., Zinga, M., Kweka, E. J., Heukelbach, J. (2010a). Knowledge, Attitudes, and Practices about Malaria and Its Control in Rural Northwest Tanzania. Malaria Research and Treatment. doi:10.4061/2010/794261

Mazigo, H. D., Obasy, E., Mauka, W., Manyiri, P., Zinga, M., Kweka, E. J., Heukelbach, J. (2010b). Knowledge, Attitudes, and Practices about Malaria and Its Control in Rural Northwest Tanzania. Malaria Research and Treatment, 2010, 794261. doi:10.4061/2010/794261

Opare, J. K. L., Antobre-Boateng, A., Afari, E., Wurapa, F., Abankwah, J., Kyeiwa-Asante, D., Serwaa, O. (2014). Community Knowledge and Perceptions on Malaria and Its Prevention and Control in the Akwapim North Municipality. Ghana. International Journal of Tropical Disease and Health, 4(1), 82-93.

President's Malaria Initiative: Malaria Operational Plan ( MOP ) Rwanda FY 2013. (2013).

Rulisa, S., Kateera, F., Bizimana, J. P., Agaba, S., Dukuzumuremyi, J., Baas, L., de Vries, P. J. (2013). Malaria prevalence, spatial clustering and risk factors in a low endemic area of Eastern Rwanda: a cross sectional study. PloS One, 8(7), e69443. doi:10.1371/journal.pone.0069443

Spjeldnæs, A. O., Kitua, A. Y., \& Blomberg, B. (2014). Education and knowledge helps combating malaria, but not degedege : a cross-sectional study in Rufiji, Tanzania. Malaria Journal, 13(200), 1-10. doi:10.1186/1475-2875-13-200

WHO. (2012). World Malaria Report 2012, (December).

WHO. (2013). World Malaria report. Nature. doi:10.1038/ nature.2013.13535 